\title{
P01-208
}

\section{PROTOCADHERIN ALPHA GENE POLYMORPHISMS IN BIPOLAR DISORDER. RESULTS FROM THE CZECH COHORT}

T. Novak ${ }^{1}$, P. Stopkova ${ }^{1}$, E. Pedrosa ${ }^{2}$, H. Lachman ${ }^{2}$

${ }^{1}$ Prague Psychiatric Center, Prague, Czech Republic, ${ }^{2}$ Department of Psychiatry and Behavioral Science, Albert Einstein College of Medicine, New York, USA

Aims: Protocadherins (PCHD) are cell adhesion proteins with an important role in neuronal migration, differentiation and synaptogenesis. The linkage studies suggest that the $5 q 31$-linked protocadherin family locus should be considered as potential candidate locus in schizophrenia and bipolar disorder. In this study, we focused particularly on single-nucleotide polymorphisms (SNPs) located in PCHDa enhancer. Results from the Czech cohort of patients with bipolar disorder (BD) will be presented.

Methods: Unrelated inpatients and outpatients with BD based on Schedule for Affective Disorders and Schizophrenia - Lifetime $(n=167)$ and blood bank donors as control subjects $(n=211)$ were recruited in the study. Four SNPs posted in dbSNP (rs31745, rs10036519, rs3756337 and rs59497) in the PCDHa gene enhancer were analyzed. The data sets were analysed using a case control design.

Results: In case of SNP, rs31745, a significant increase in homozygosity for the minor allele (T) was detected in patients with BD; $5 \%$ had this genotype, but no controls $(p=0.001)$. The distribution of allels did not differ between patients and controls. No significant differences were found in allele or genotype distribution for three other SNPs.

Conclusions: The findings suggest that the PCHDa is an interesting gene family to consider in BD susceptibility. 